\title{
ON A LIMIT THEOREM AND INVARIANCE PRINCIPLE FOR SYMMETRIC STATISTICS*
}

BY

\section{MANDREKAR (MICHIGAN) AND B. V. RAO (CALCUTTA)}

Abstract. The paper contains direct proofs of two important theorems. First of them (Theorem 0.6) was formulated and proved by Dynkin and Mandelbaum [2], the second one (Theorem 1.2) - by Mandelbaum and Taqqu [3].

0. Introduction. The purpose of this note is to give a direct proof of some recent important results of Dynkin and Mandelbaum [2]. This also provides immediately the results in [3] with a very simple proof. This is achieved by avoiding the use of Poisson process.

Let us set up some notation. Let $(X, \Sigma, \mu)$ be a probability space and $\left(X^{k}, \Sigma^{k}, \mu^{k}\right)$ be the $k$-fold produce probability space. Let $h_{k}\left(x_{1}, \ldots, x_{k}\right)$ be a symmetric function of $k$ variables. We call it canonical if

$$
\int h_{k}\left(x_{1}, \ldots, x_{k-1}, y\right) d \mu=0 \text { for all } x_{1}, \ldots, x_{k-1} \in X^{k-1} \text {. }
$$

Let $X_{1}, \ldots, X_{n}$ be an i.i.d. $X$-valued random variable on a probability space with distribution $\mu$. As in [2], define

$$
\sigma_{k}^{n}\left(h_{k}\right)= \begin{cases}\sum_{1 \leqslant s_{1}<\ldots<s_{k} \leqslant n} h_{k}\left(X_{s_{1}}, \ldots, X_{s_{k}}\right) & \text { for } k \leqslant n, \\ 0 & \text { for } k>n .\end{cases}
$$

Let

$$
H=\left\{\left(h_{0}, h_{1}, \ldots\right): h_{k} \text { canonical and } \sum_{k=1}^{\infty} \frac{1}{k !}\left\|h_{k}\right\|_{2}^{2}<\infty\right\},
$$

where $h_{0}$ is a constant and \|\|$_{2}$ is the norm in $L^{2}\left(X^{k}, \Sigma^{k}, \mu^{k}\right)$. On $H$ define

$$
\|h\|^{2}=\sum_{k=0}^{\infty}\left\|h_{k}\right\|_{2}^{2} / k !
$$

$H$ is the so-called exponential (Foch) space of $L_{0}^{2}(X, \Sigma, \mu)\left(\varphi \in L^{2}(X, \Sigma, \mu)\right.$ with $E \varphi(X)=0)$. It is a Hilbert space under coordinate addition, scalar

* This research was supported by ONR N00014-85-K-0150 and the Air Force Office of Scientific Research Contract No. F 49620 85C 0144. 
multiplication and \|\| . For each $\varphi \in L_{0}^{2}(X, F, \mu), h^{\varphi} \in H$ with $h_{k}^{\varphi}=\varphi\left(x_{1}\right), \ldots, \varphi\left(x_{k}\right)$. It can be easily seen that $\operatorname{sp}\left\{h^{\varphi}: \varphi \in L_{0}^{2}(X, F, \mu)\right\}$ is dense in $H$. Define, for each $h \in H$,

$$
Y_{n}(h)=\sum_{k=0}^{\infty} n^{-k / 2} \sigma_{k}^{n}\left(h_{k}\right) .
$$

Since $\sigma_{k}^{n}\left(h_{k}\right)=0$ for $k>n$, this is a finite sum. Also, let

$$
Y_{n}^{t}(h)=\sum_{k=0}^{\infty} n^{-k / 2} \sigma_{k}^{[n t]}\left(h_{k}\right)
$$

The main purpose is to show directly that

$$
Y_{n}(h) \stackrel{D}{\rightarrow} \sum_{k=0}^{\infty} \frac{I_{k}\left(h_{k}\right)}{k !},
$$

where $\stackrel{D}{\rightarrow}$ denotes convergence in distribution and $I_{k}\left(h_{k}\right)$ denotes Ito-Wiener multiple integral of $h_{k}$ with respect to Gaussian random measure $W$ with $E W(A) W\left(A^{\prime}\right)=\mu\left(A \cap A^{\prime}\right)$.

In the next section we discuss the convergence of $Y_{n}^{t}(h)$. We observe that for $\varphi \in L_{0}^{2}(X, \Sigma, \mu)$

$$
\begin{aligned}
Y_{n}\left(h^{\varphi}\right) & =\sum_{k=0}^{n} n^{-k / 2} \sum_{1 \leqslant s_{1}<\ldots<s_{k} \leqslant n} \varphi\left(X_{s_{1}}\right) \ldots \varphi\left(X_{s_{k}}\right) \\
& =\sum_{k=0}^{n} \sum_{1 \leqslant s_{1}<\ldots<s_{k} \leqslant n} \frac{\varphi\left(X_{s_{1}}\right)}{\sqrt{n}} \ldots \frac{\varphi\left(X_{s_{k}}\right)}{\sqrt{n}}=\prod_{1}^{n}\left(1+\frac{\varphi\left(X_{i}\right)}{\sqrt{n}}\right) .
\end{aligned}
$$

Let us observe that, for any $\varepsilon>0$,

$$
\sum_{j} P\left(\left|\varphi\left(X_{j}\right)\right|>\sqrt{\varepsilon j}\right)=\sum_{j} P\left(\left|\varphi\left(X_{1}\right)\right|^{2}>\varepsilon j\right) \leqslant C\|\varphi\|_{2}^{2}<\infty .
$$

Hence by Borel-Cantelli lemma, a.s. (for $j \leqslant n)\left|\varphi\left(X_{j}\right)\right| \leqslant \sqrt{\varepsilon j} \leqslant \sqrt{\varepsilon} \sqrt{n}$ for $j \geqslant$ some $N(\omega)(N(\omega)<\infty)$. But

$$
\prod_{1}^{n}\left(1+\frac{\varphi\left(X_{j}\right)}{\sqrt{n}}\right)=\prod_{1}^{N(\omega)}\left(1+\frac{\varphi\left(X_{j}\right)}{\sqrt{n}}\right) \prod_{N(\omega)}^{n}\left(1+\frac{\varphi\left(X_{j}\right)}{\sqrt{n}}\right)
$$

giving for a.s. $w$, so

$$
\lim _{n} Y_{n}\left(h^{\varphi}\right)=\lim _{n} \prod_{N(\omega)}^{n}\left(1+\frac{\varphi\left(X_{j}\right)}{\sqrt{n}}\right) .
$$
and

Thus WLOG, we can assume for $n$ large $\left|\varphi\left(X_{j}\right) / \sqrt{n}\right|<1$ a.s. for all $j \leqslant n$

$$
Y_{n}\left(h^{\varphi}\right)=\prod_{1}^{n}\left(1+\frac{\varphi\left(X_{j}\right)}{\sqrt{n}}\right) \text {. }
$$


Taking $\log$ on both sides and expanding $\log (1+x)$ we have

$$
\log \prod_{1}^{n}\left(1+\frac{\varphi\left(X_{j}\right)}{\sqrt{n}}\right)=\sum_{1}^{n} \frac{\varphi\left(X_{j}\right)}{\sqrt{n}}-\frac{1}{2} \sum_{1}^{n} \frac{\varphi\left(X_{j}\right)^{2}}{n}+\varepsilon_{n}(\varphi),
$$

where $\varepsilon_{n}(\varphi) \stackrel{P}{\rightarrow} 0$ by the WLLN and since $\max \left|\varphi\left(X_{j}\right) / \sqrt{n}\right| \stackrel{P}{\rightarrow} 0$ by Chebychev's Inequality, i.e. the $\left(Y_{n}\left(h^{\varphi}\right)\right) \stackrel{D}{\rightarrow} \exp \left[I_{1}(\varphi)-\frac{1}{2}\|\varphi\|_{2}^{2}\right]$. Using Cramér-Wold device and the above argument we get

(0.3) LemMa. For any finite subset $\left\{\varphi_{1} \ldots \varphi_{k}\right\} \subseteq L^{2}(X, \Sigma, \mu)$

$$
\left(Y_{n}\left(h^{\varphi_{1}}\right), \ldots, Y_{n}\left(h^{\varphi_{k}}\right)\right) \stackrel{D}{\rightarrow}\left(\exp \left(I_{1}\left(\varphi_{1}\right)\right)-\frac{1}{2}\left\|\varphi_{1}\right\|_{2}^{2}, \ldots, \exp \left(I_{1}\left(\varphi_{k}\right)-\frac{1}{2}\left\|\varphi_{k}\right\|_{2}^{2}\right) .\right.
$$

As a consequence, we get for $\left\{\varphi_{i}, i \in I\right\}$ a finite subset of $L^{2}(X, \Sigma, \mu)$ and $\left\{c_{i}, i \in I\right\} \subseteq \boldsymbol{R}$,

$$
Y_{n}\left(\sum_{i \in I} c_{i} h^{\varphi_{i}}\right) \stackrel{D}{\rightarrow} \sum_{k=0}^{\infty} \frac{I_{k}\left(\left[\sum_{i \in I} c_{i} h^{\varphi_{i}}\right]_{k}\right)}{k !} .
$$

We now observe that for $h, h^{\prime} \in H$,

$$
E\left[Y_{n}(h)-Y_{n}\left(h^{\prime}\right)\right]^{2}=\sum_{k}\left(\begin{array}{l}
n \\
k
\end{array}\right) n^{-k}\left\|h_{k}-h_{k}^{\prime}\right\|^{2} \leqslant E\left\|h-h^{\prime}\right\|^{2},
$$

since $E \sigma_{k}^{n}\left(h_{k}-h_{k}^{\prime}\right) \sigma_{l}^{n}\left(h_{l}-h_{l}^{\prime}\right)=\left(\begin{array}{l}n \\ k\end{array}\right)\left\|h_{k}-h_{k}^{\prime}\right\|^{2} \delta_{k l}$ (by [2], p. 744). Also,

$$
E\left(\sum_{k=0}^{\infty} I_{k}\left(h_{k}\right) / k !-\sum_{k=0}^{\infty} \frac{I_{k}\left(h_{k}^{\prime}\right)}{k !}\right)^{2}=\left\|h-h^{\prime}\right\|^{2}
$$

Thus we get

(0.6) TheOREM. For any $h \in H$,

$$
Y_{n}(h) \stackrel{D}{\rightarrow} W(h)=\sum_{k=0}^{\infty} \frac{I_{k}\left(h_{k}\right)}{k !} .
$$

Proof. Let $h \in H$ and $\varepsilon>0$. Choose

$$
h^{\prime}=\sum_{i \in I} c_{i} h^{\varphi_{i}}
$$

such that $\left\|h-h^{\prime}\right\|^{2}<\varepsilon / 2$. Now consider, for $t \in \boldsymbol{R}$,

$$
\left|E\left(e^{i t Y_{n}(h)}-e^{i t W(h)}\right)\right| \leqslant E\left|e^{i t Y_{n}(h)}-e^{i t Y_{n}\left(h^{\prime}\right)}\right|+E\left|e^{i t Y_{n}\left(h^{\prime}\right)}-e^{i t W\left(h^{\prime}\right)}\right|+E\left|e^{i t W\left(h^{\prime}\right)}-e^{i t W(h)}\right| .
$$

Using Schwartz's Inequality and the fact $\left|e^{i x}-1\right| \leqslant|x|$, we get that the first and third terms of the above inequality are dominated by $t^{2} E\left\|h-h^{\prime}\right\|^{2}$ using $(0.4)$ and $(0.5)$. Hence, by $(0.3)^{\prime}$, 


$$
\varlimsup \overline{\lim }\left|E e^{i t Y_{n}(h)}-E e^{i t W(h)}\right| \leqslant \varepsilon / 2 .
$$

As $\varepsilon$ is arbitrary, we get the result.

Finally, we make some observations to be used later:

(0.7) $Y_{n}^{t}\left(h^{\varphi}\right)=\sum_{k=0}^{[n t]} n^{-k / 2} \sum_{1 \leqslant s_{1}<\ldots<s_{k} \leqslant[n t]} \varphi\left(X_{s_{1}}\right) \ldots \varphi\left(X_{s_{k}}\right)=\prod_{1}^{[n t]}\left(1+\frac{\varphi\left(X_{i}\right)}{\sqrt{n}}\right)$.

Also, $\min (t, s) \mu\left(A \cap A^{\prime}\right)$ is a covariance on $[0, \infty) \times \Sigma$ giving that there exists a centered Gaussian process $W(t, A)$ with $E W(t, A) W\left(s, A^{\prime}\right)=$ $\min (t, s) \mu\left(A \cap A^{\prime}\right)$. Let, for $T<\infty$,

$$
H_{T}=\left\{\left(h_{0}, h_{1}, \ldots,\right) \in H: \sum_{k=0} T^{k} \frac{\left\|h_{k}\right\|^{2}}{k !}<\infty\right\} .
$$

1. Invariance Principle. Let $D[0, T], T \leqslant \infty$, be the space of right continuous functions on $[0, \mathrm{~T}]([0, \infty))$ with left limits at each $t \leqslant T$. The space $D[0, T]$ is endowed with Skorohod topology [1]. The topology in $D[0, \infty)$ is the one described in Whitt [4]. We note that

$$
X_{[n t]}=\sum_{1}^{[n t]}\left(\frac{\varphi^{2}\left(X_{i}\right)-E \varphi^{2}}{n}\right)
$$

has stationary independent increments. So, for $\varepsilon>0$,

$$
P\left(\sup _{0 \leqslant t \leqslant T}\left|X_{[n t]}\right|>\varepsilon\right) \leqslant C P\left(\left|X_{[n T]}\right| \geqslant \varepsilon\right) \rightarrow 0
$$

by the weak law of large numbers. Using this, the arguments preceding Lemma 0.3 , invariance principle and Cramér-Wold device we get the following analogue of Lemma 0.3:

LEMMA 1.1. $\left(Y_{n}^{t}\left(h^{\varphi_{1}}\right), \ldots, Y_{n}^{t}\left(h^{\varphi_{k}}\right)\right) \stackrel{D_{k, 7}}{\longrightarrow}\left(\exp \left(I_{1}^{t}\left(\varphi_{j}\right)-\frac{1}{2} t\left\|\varphi_{j}\right\|^{2}\right), j=1, \ldots, k\right)$, where $I^{t}\left(\varphi_{j}\right)=\iint 1_{(0, t]}(u) \varphi_{j}(x) W_{k}(d u, d x)$.

Here $\stackrel{D_{k, T}}{\longrightarrow}$ denotes convergence in $D^{k}[0, T]$ with respect to product topology.

We note that $W(t, A)$ is a Brownian motion for each $A \in \Sigma$. Thus we can choose $I^{t}(\varphi)$ continuous for each $\varphi$ and a martingale in $t$ as $I^{t}(\varphi)=\int \varphi(x) W(t, d x)$. We get, for $\left\{c_{1}, \ldots, c_{k}\right\} \subseteq \boldsymbol{R}$ ( $k$ finite),

$$
Y^{t}\left(\sum_{j=1}^{k} c_{j} h^{\varphi_{j}}\right) \rightarrow \sum_{j=1}^{k} c_{j} \exp \left(I^{t}\left(\varphi_{j}\right)-\frac{1}{2} t\left\|\varphi_{j}\right\|^{2}\right) \text {. }
$$

Let $\varphi \in L_{0}^{2}(X, \Sigma, \mu),\|\varphi\|=1$, and write

$$
\left(\varphi^{k}\right)^{t}=\varphi\left(x_{1}\right) \ldots \varphi\left(x_{k}\right) 1_{(0, t]}\left(u_{1}\right) \ldots 1_{(0, t]}\left(u_{k}\right) .
$$

Define $I_{k}\left(\varphi^{k}\right)^{t}=k ! H_{k}(t, I(\varphi))$, where $H_{k}$ is Hermite polynomial, i.e.

$$
\sum_{k=0}^{\infty} \gamma^{k} H_{k}(t, x)=\exp \left(\gamma x-\frac{1}{2} \gamma^{2} t\right)
$$


For $\varphi \in L_{0}^{2}(X, \Sigma, \mu),\|\varphi\|=1$, we define for $\left(\left(h^{\varphi}\right)^{t}=\left(1, \varphi^{t},\left(\varphi^{2}\right)^{t}, \ldots\right)\right.$,

$$
W\left(h^{\phi}\right)^{t}=\sum_{k=0}^{\infty} \frac{I_{k}\left(\varphi^{k}\right)^{t}}{k !}
$$

and extend it linearly to $\left(\Sigma c_{j}\left(h^{\varphi_{j}}\right)^{t}\right)$. It is a martingale.

Let $h \in H_{T},\{h(n)\}$ a sequence in sp $\left\{\left(h^{\varphi}\right)^{t}, \varphi\right.$ in CONS in $\left.L_{0}^{2}(X, \Sigma, \mu)\right\} \subseteq H_{T}$; then

$$
\begin{aligned}
P\left(\sup _{t \leqslant T}\left|W^{t}(h(n)-h(m))\right| \geqslant \varepsilon\right) \leqslant E\left|W^{T}(h(m)-h(n))\right|^{2} & \\
& =\sum_{k=0}^{\infty} T^{k} \frac{\left\|h_{k}(m)-h_{k}(n)\right\|^{2}}{k !},
\end{aligned}
$$

using Doob's inequality and argument as in (0.5). Define, for $h \in H^{t}$, $W^{t}(h)=-\lim W^{t}\left(h_{n}\right)$, where the limit is uniform on compact for $h_{n} \rightarrow h$. Then $W^{t}(h)$ is right continuous martingale and has the same distribution as $\Sigma_{k} I_{k}^{t}\left(h_{k}\right) / k$ !. Now we derive the main theorem of [3].

THEOREM 1.2. $Y_{n}^{t}(h) \stackrel{D}{\rightarrow} W^{t}(h)$ in $D[0, T]$ for $h \in H^{T}$ for each $T<\infty$.

Proof. Let $h \in H$ and $\varepsilon>0$, choose $h_{k}^{\prime} \in \operatorname{sp}\left\{h^{\varphi}: \varphi \in L_{0}^{2}(X, \Sigma, \mu)\right\} \ni h_{k} \rightarrow h$.

Now define $X_{n k}=Y_{n}^{\prime}\left(h_{k}^{\prime}\right), Z_{\dot{n}}=Y_{\dot{n}}(h), X_{\dot{k}}=W^{\prime}\left(h_{k}^{\prime}\right)$ and $X=W \cdot(h)$. Then $X_{\dot{n}, k} \stackrel{D}{\rightarrow} X_{\dot{k}}$ as $n \rightarrow \infty$ in $D[0, T]$ for each $T<\infty$ by Lemma 1.1. Also $X_{\dot{k}} \stackrel{D}{\rightarrow} X$ as $n \rightarrow \infty$ in $D[0, T]$ for each $T<\infty$. In addition,

$$
P\left(\sup _{0 \leqslant t \leqslant T}\left|X_{\dot{n} k}-Z_{\dot{n}}\right| \geqslant \varepsilon\right) \leqslant E\left|Y_{n}^{T}\left(h-h_{k}^{\prime}\right)\right|^{2} \leqslant T\left\|h-h_{k}^{\prime}\right\|
$$

giving

$$
\lim _{k \rightarrow \infty} \varlimsup_{n} P\left(\varrho\left(X_{n k}, Z_{n}\right) \geqslant \varepsilon\right) \rightarrow 0
$$

with $\varrho$ being the Skorohod metric on $D[0, T]$. This implies (by [1], Thm 4.2, p. 25) that $Z_{\dot{n}} \stackrel{D}{\rightarrow} W \cdot(h)$ in $D[0, T](T<\infty)$ giving the result.

Remark. In the above arguments we may use an interpolated version of $Y_{n}^{t}(h)$ from the beginning and use appropriate version of Donsker's Invariance Principle to conclude above convergence occurs in $D[0, T]$ in sup norm giving $W^{t}(h)$ continuous.

\section{REFERENCES}

[1] P. Billingsley, Convergence of probability measures, Wiley, New York 1968.

[2] E. B. Dynkin and A. Mandelbaum, Symmetric statistics, Poisson point processes and multiple Wiener integrals, Ann. Statist. 11 (1983), p. 739-745. 
[3] A. Mandelbaum and M. S. Taqqu, Invariance principles for symmetric statistics, Ann. Statist. 12 (1984), p. 483-496.

[4] W. Whitt, Some useful functions for functional limit theorems, Math. Op. Research 5 (1980), p. $67-85$.

\section{Michigan State University}

and

Center for Stochastic Processes
Indian Statistical Institute

Calcutta India

Received on 13. 4. 1987 